\title{
Intermedin protects against myocardial ischemia-reperfusion injury in hyperlipidemia rats
}

\author{
S.M. Yang ${ }^{1}$, J. Liu ${ }^{2}$ and C.X. Li ${ }^{1}$ \\ ${ }^{1}$ Department of Cardiac Surgery, Affiliated Hospital of Medical College, \\ Qingdao University, Qingdao, Shandong, China \\ ${ }^{2}$ Department of Library Management, Affiliated Hospital of Medical College, \\ Qingdao University, Qingdao, Shandong, China \\ Corresponding author: S.M. Yang \\ E-mail: smjlcn@163.com
}

Genet. Mol. Res. 13 (4): 8309-8319 (2014)

Received July 26, 2013

Accepted February 10, 2014

Published October 20, 2014

DOI http://dx.doi.org/10.4238/2014.October.20.7

\begin{abstract}
Hyperlipidemia is a well-established risk factor for the development of coronary atherosclerosis, while intermedin (IMD) has been identified as a novel calcitonin/calcitonin gene-related peptide family member involved in cardiovascular protection. However, whether IMD protects against hyperlipidemia-associated myocardial ischemia/reperfusion $(\mathrm{MI} / \mathrm{R})$ injury is unknown. We established a hyperlipidemia model using Sprague-Dawley rats, and created a MI/R condition by ligating the cardiac left circumflex artery. The possible pathophysiological role of IMD and its physiological function in $\mathrm{MI} / \mathrm{R}$ was further studied. The level of IMD significantly decreased in hyperlipidemia rats $(\mathrm{P}<0.05)$. After $\mathrm{MI} / \mathrm{R}$, the IMD level was increased both in the plasma and myocardial tissue of hyperlipidemia rats compared to the sham-operated rats $(\mathrm{P}<0.001)$. As evaluated by the activity of LDH, CK-MB, MDA and SOD, additional IMD was revealed to alleviate $\mathrm{MI} / \mathrm{R}$ heart injury in hyperlipidemia rats $(\mathrm{P}<0.05)$. By regulating the process of cardiomyocyte apoptosis
\end{abstract}


and inflammatory reaction, IMD could perform an important role in cardio-protection, especially against hyperlipidemia-associated MI/R injury. Additional IMD could protect cardiac myocytes against $\mathrm{MI} / \mathrm{R}$ injury via reduction of apoptosis and inflammation in the hyperlipidemia rat model, and thus, it may play a potential role as a novel therapeutic target for cardiac ischemic injury in hyperlipidemic patients.

Key words: Hyperlipidemia; Myocardial; Ischemia-reperfusion injury; Intermedin; Cardiovascular protection; Rat

\section{INTRODUCTION}

Hyperlipidemia, characterized by elevated serum lipoproteins, total cholesterol (TC) and triglycerides (TG), is associated with an increased risk of coronary heart disease such as myocardial infarction and atherosclerosis (Ross and Harker, 1976; Assmann and Schulte, 1992). Several studies conducted in rodent models have suggested that myocardial ischemia/reperfusion (MI/R) injury in the setting of hyperlipidemia may result in more myocardial necrosis and apoptosis compared with non-hyperlipidemia animals (Wang et al., 2002; Osipov et al., 2009). For patients with a first MI condition, hyperlipidemia has been reported to have a detrimental effect on ventricular function with a more pronounced deterioration; but if treated with lipid-lowering agents before MI, myocardial infarct size can be significantly reduced both in patients and experimental animals (Wang et al., 1998; Aronow et al., 2008).

However, pharmacologic agents for reducing myocardial injury in hyperlipidemia patients are limited; thus, newly effective drugs need to be further explored. Intermedin (IMD), also known as adrenomedullin 2, is a newly discovered peptide that belongs to the calcitonin gene-related peptide (CGRP) superfamily (Roh et al., 2004). IMD is expressed in many organs of body, including those of the gastrointestinal tract, pancreas and lung, and it has been shown to be a multifunctional peptide involved in the regulation of cardiovascular dynamics, including potently dilating systemic and pulmonary vessels, influencing regional blood flow, augmenting cardiac contractility, and increasing urinary flow and urinary sodium excretion (Chang et al., 2004; Bell and McDermott, 2008; Smith et al., 2009). The distribution of IMD in the cardiovascular system is compatible with a physiological role in the inhibition of collagen synthesis, which simultaneously attenuates cardiac fibroblast proliferation and cardiomyocyte hypertrophy (Pan et al., 2005; Yang et al., 2009). In recent studies, increased attention has been devoted to the role of endogenous IMD in cardiovascular diseases. The robust IMD expression in a sick cardiovascular system implies a significant effect on cardiovascular system pathology (Bell and McDermott, 2008; Bell et al., 2008). Additionally, IMD has been reported to reduce hyperglycemia-exacerbated MI/R injury in diabetic rat models ( $\mathrm{Li}$ et al., 2013). As a major cause of myocardial infarction similar to diabetes, however, whether the condition of hyperlipidemia can be alleviated by IMD is still unknown.

In the current study, we aimed to determine whether IMD can protect the heart with hyperlipidemia against MI/R injury and, if so, to explore further its possible mechanism. 


\section{MATERIAL AND METHODS}

\section{Animals and interventions}

The male Sprague-Dawley (SD) rats (weight: 250-350 g) were purchased from Qingdao Drug Administration, China. Rats were housed in a specific pathogen-free room, with controlled temperature $\left(24^{\circ} \pm 2^{\circ} \mathrm{C}\right)$ and humidity $(55 \pm 10 \%)$ and free access to standard rat chow and water in the experiment center of the Affiliated Hospital of Medical College, Qingdao University. The ordinary diet and high-fat diet (HFD) (consisting of 10\% lard oil, $2 \%$ cholesterol, and $88 \%$ standard diet) (Liu et al., 2013) were obtained from Shanghai Si-LaiKe Experimental Animal Ltd. All study protocols were approved by the Medical College of Qingdao University Animal Care Committee. Rats were randomly assigned to five different groups: non-hyperlipidemia group $(\mathrm{NH}, \mathrm{N}=10$, non-hyperlipidemia + ischemia/reperfusion (NIR, $\mathrm{N}=10$ ), hyperlipidemia group $(\mathrm{HG}, \mathrm{N}=10$ ), hyperlipidemia + ischemia/reperfusion (HIR, $\mathrm{N}=10$ ), and hyperlipidemia + ischemia/reperfusion + IMD treatment (IMD, N = 10). In the IMD treatment group, IMD $(20 \mathrm{nmol} / \mathrm{kg})$ was diluted in $1 \mathrm{X}$ phosphate-buffered saline and infused $20 \mathrm{~min}$ after MI onset, via the left femoral vein over a period of $10 \mathrm{~min}$ (Du et al., 2011).

\section{$\mathbf{M I} / \mathbf{R}$}

Five weeks after the initial treatment, SD rats were anesthetized by intraperitoneal injection of $7 \%$ chloral hydrate (Aoxin, China). A left thoracotomy and pericardiotomy were performed. The left coronary artery was dissected above the first diagonal branch and ligated immediately proximal to the left circumflex arterial origin with silk thread. Slipknot-induced occlusion proceeded for $30 \mathrm{~min}$. R wave amplification and ST segment depression were observed immediately in lead II of the attached electrocardiogram. Myocardium distal to the ligation line darkened, indicating myocardial ischemia. After $30 \mathrm{~min} \mathrm{MI}$, the slipknot was released for $120 \mathrm{~min}$, allowing reperfusion (R) (Fang et al., 2009). Blood was collected after $\mathrm{R}$ by cardiac puncture and centrifuged at $2000 \mathrm{~g}$ for $10 \mathrm{~min}$. Serum and plasma were stored at $-80^{\circ} \mathrm{C}$ for further analysis. Rats were sacrificed via direct intraventricular $\mathrm{KCl}$ injection (Gao et al., 2011). Hearts were removed and rinsed with ice-cold phosphate buffered saline. Ventricular tissue was immediately frozen in liquid nitrogen and stored at $-80^{\circ} \mathrm{C}$.

\section{Determination of plasma IMD levels}

Rats' blood samples were prepared as previously described (Zhang et al., 2009). Plasma was loaded to a Sep-Pak C18 cartridge (Phoenix, AZ, USA) and pre-equilibrated with 0.5 $\mathrm{mM}$ acetic acid (Xilong, China). The adsorbed material was eluted with $4 \mathrm{~mL} 50 \% \mathrm{CH}_{3} \mathrm{CN}$ (Saike, China) containing 0.1\% trifluoroacetic acid (Guochen, China) (Jiang et al., 2004). After lyophilization, the residue was dissolved in radioimmunoassay buffer and analyzed following the IMD kit's manufacturer protocol directions (Phoenix, AZ, USA).

\section{Serum biochemical analysis}

The serum concentrations of TG, TC LDL-C, and HDL-C were measured accord- 
ing to the manufacturer instructions (Nanjing Jiancheng Bioengineering, China). The lipid concentrations in each sample were calculated using standards provided in the kits, taking into account any dilution factors required. For determining the serum level of LDH, CK-MB, tumor necrosis factor alpha (TNF- $\alpha$ ) and interleukin-6 (IL-6), corresponding rat ELISA kits were used in accordance with the manufacturer protocol (Nanjing Jiancheng Bioengineering).

\section{Cardiac tissue biochemical measurement}

Cardiac malondialdehyde (MDA), superoxide dismutase (SOD), TNF- $\alpha$ and IL-6 levels were determined using the tissue samples from the left ventricular apex ischemic region after the 2-h reperfusion period and homogenized in $1.15 \% \mathrm{KCl}$ (Guochen, China) buffer (Zhang et al., 2012). Myocardial MDA, SOD, TNF- $\alpha$ and IL-6 content were detected per manufacturer protocol (Nanjing Jiancheng Bioengineering, CA, USA).

\section{Western blot analysis}

Frozen ventricle samples were homogenized in protein lysate buffer $[50 \mathrm{mM}$ Tris$\mathrm{HCl}$, pH 7.5, $50 \mathrm{mM}$ 2-mercaptoethanol, $5 \mathrm{mM}$ EGTA, 2 mM EDTA, 1\% NP-40, 0.1\% sodium dodecyl sulfate polyacrylamide (SDS), $0.5 \%$ deoxycholic acid, $10 \mathrm{mM} \mathrm{NaF}, 1 \mathrm{mM}$ PMSF, $25 \mathrm{mg} / \mathrm{mL}$ leupeptin, $2 \mathrm{mg} / \mathrm{mL}$ aprotinin] (CWBIO, China), and protein concentrations were determined using the BJKO assay system (BJKO, China). Total proteins were fractionated using SDS gel electrophoresis and transferred to a polyvinylidene fluoride membrane. Primary antibodies (Abcam, UK; CWBIO, China) were diluted 1:1000 in TBST (CWBIO, China), added to the membrane, and incubated overnight with agitation at room temperature. After incubation with horseradish peroxidase (HRP)-conjugated goat anti-rabbit IgG (CWBIO, China) at room temperature for $60 \mathrm{~min}$, signals were detected using the VILBER enhanced chemiluminescence system (VILBER LOURMAT, France) according to manufacturer instructions.

\section{Statistical analysis}

All values are reported as means $\pm \mathrm{SE}$. We evaluated significant differences for in vitro data using the Student $t$-test. $\mathrm{P}<0.05$ was considered to be statistically significant for all tests.

\section{RESULTS}

\section{HFD treatment successfully induced a hyperlipidemia model}

Blood lipid levels of the rats were recorded at the beginning of the experiment and each week after initial HFD treatment. In the initial period, no significant difference was found in blood lipids between all groups (Table 1). Five weeks after HFD treatment, hyperlipidemia rats manifested significantly increased blood TG, TC, LDL-C levels and decreased levels of HDL-C compared with the non-hyperlipidemia group $(\mathrm{P}<0.05$, Table 1$)$, indicating that a hyperlipidemia rat model had been successfully induced (Figure 1A). 
Intermedin protects against reperfusion injury

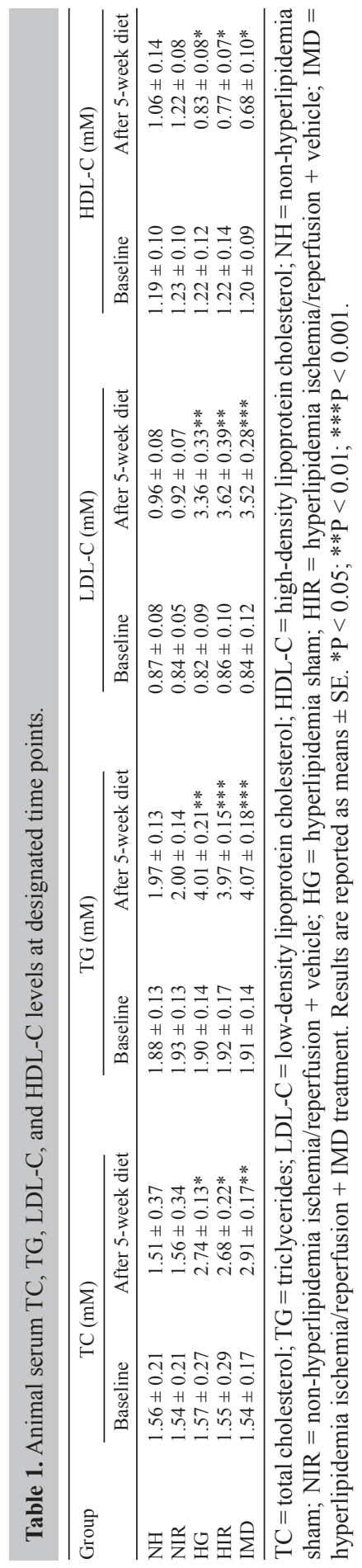


A

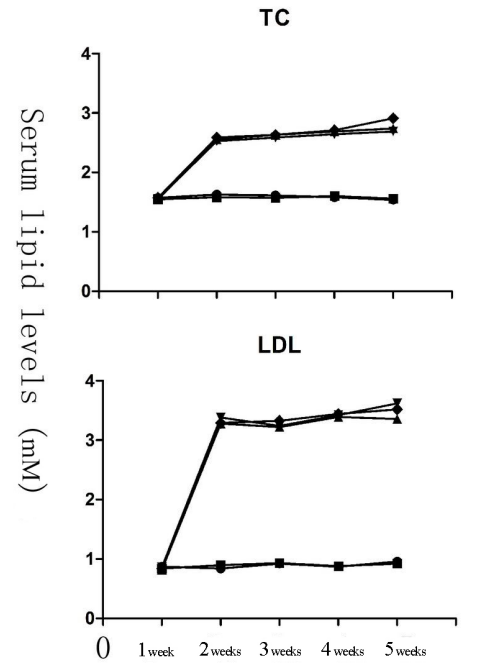

B

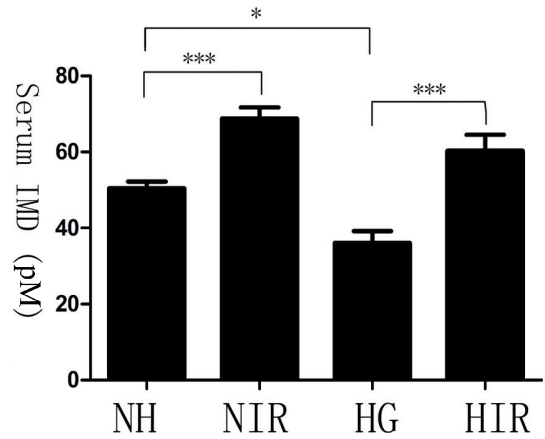

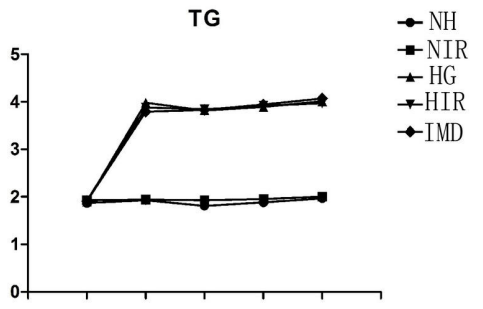

HDL

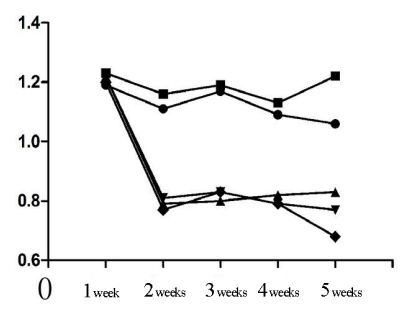

C

IMD

GAPDH
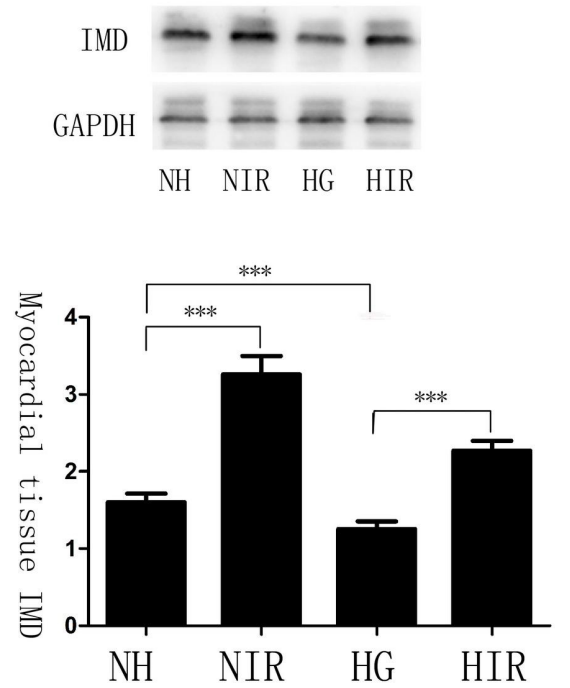

Figure 1. Intermedin (IMD) levels in normal and hyperlipidemia rats in response to MI/R. A. Animal blood TC, TG, LDL-C, and HDL-C levels at designated time points. B. IMD levels in hyperlipidemia rat plasma. C. Western blot demonstrating altered IMD expression in myocardial tissue. Results were shown by three dependent experiments. $\mathrm{NH}=$ non-hyperlipidemia sham; NIR = non-hyperlipidemia ischemia/reperfusion + vehicle; $\mathrm{HG}=$ hyperlipidemia sham; HIR = hyperlipidemia ischemia/reperfusion + vehicle; IMD = hyperlipidemia ischemia/reperfusion + IMD treatment. Results are reported as means \pm SE. $* \mathrm{P}<0.05 ; * * * \mathrm{P}<0.001$ significant. For other abbreviations, see legend to Table 1 .

\section{Hyperlipidemia rats manifested significantly decreased IMD levels, while MI/R increased IMD levels}

The IMD levels determined are shown in Figure 1. It was not only the plasma but also 
myocardial tissue manifested significantly decreased IMD levels in hyperlipidemia rats compared to control animals $(\mathrm{P}<0.05)$. After $\mathrm{MI} / \mathrm{R}$, the IMD levels both in plasma and myocardial tissue were increased in the hyperlipidemia and non-hyperlipidemia rats compared with the sham-operated rats $(\mathrm{P}<0.001$, Figure $1 \mathrm{~B}$ and $\mathrm{C})$, suggesting that $\mathrm{MI} / \mathrm{R}$ could increase IMD levels.

\section{IMD attenuated MI/R injury in hyperlipidemia animals}

To determine the state of myocardial injury in the animals, we examined some biochemical parameters after MI/R. As a result, significantly increased levels of LDH, CK-MB and MDA and decreased activity of SOD was observed both in the normal and hyperlipidemia rats as a response to $\mathrm{MI} / \mathrm{R}(\mathrm{P}<0.05$, Table 2$)$. Conversely, with IMD administration, a significant reduction was found in serum LDH and CK-MB and myocardial MDA, whereas the SOD levels was simultaneously increased by $39 \%$ compared with no IMD injection $\mathrm{MI} / \mathrm{R}$ rats $(\mathrm{P}<0.05$, Table 2$)$. All these data suggested that IMD may have protective roles in MI/R injury for the hyperlipidemia animals.

Table 2. Intermedin (IMD) decreases myocardial injury in hyperlipidemia animals.
\begin{tabular}{lccccc}
\hline Marker/Group & NH & NIR & HG & HIR & IMD \\
\hline LDH (U/L) & $5157.89 \pm 312.08$ & $6744.79 \pm 324.88^{*}$ & $5938.24 \pm 347.53$ & $9663.16 \pm 475.97^{\Delta}$ & $6845.37 \pm 498.31^{\star}$ \\
CK-MB (U/L) & $1374.83 \pm 348.21$ & $2525.50 \pm 303.49^{*}$ & $1797.17 \pm 225.22$ & $3770.50 \pm 434.95^{\Delta}$ & $2836.86 \pm 275.18^{\Delta}$ \\
MDA (nmol/mg) & $4.28 \pm 0.66$ & $9.51 \pm 1.97^{*}$ & $6.43 \pm 1.02$ & $13.58 \pm 3.72^{\Delta}$ & $9.59 \pm 1.63^{\Delta}$ \\
SOD (U/mg) & $108.69 \pm 21.03$ & $63.86 \pm 13.78^{*}$ & $91.05 \pm 12.79$ & $52.94 \pm 12.56^{\Delta}$ & $73.59 \pm 11.38^{\Delta}$ \\
\hline
\end{tabular}

$\mathrm{NH}=$ non-hyperlipidemia sham; NIR = non-hyperlipidemia ischemia/reperfusion + vehicle; $\mathrm{HG}=$ hyperlipidemia sham; HIR = hyperlipidemia ischemia/reperfusion + vehicle; IMD $=$ hyperlipidemia ischemia/reperfusion + IMD treatment. $\mathrm{LDH}=$ lactate dehydrogenase; $\mathrm{CK}-\mathrm{MB}=$ creatine kinase-MB; $\mathrm{MDA}=$ malondialdehyde; $\mathrm{SOD}=$ superoxide dismutase. Results are reported as means $\pm \mathrm{SE} .{ }^{*} \mathrm{P}<0.05 v s \mathrm{NH},{ }^{\Delta} \mathrm{P}<0.05$ vs $\mathrm{HG},{ }^{\Delta} \mathrm{P}<0.05$ vs HIR. $\mathrm{N}=10$ /group.

\section{IMD attenuated cardiomyocyte apoptosis in hyperlipidemia rats after MI/R}

It has been previously established that the expression of caspase-3, Bax and Bcl-2 has crucial roles in the progression of cell apoptosis (Babu et al., 2004; Song et al., 2012). To determine whether IMD protection in hyperlipidemia rats is associated with apoptosis inhibition, we examined the expression levels of caspase-3, Bax and Bcl-2, by Western blot analysis. Consequently, after MI/R, caspase-3 and Bax proteins were significantly upregulated both in hyperlipidemia and normal rats, whereas the expression of Bcl-2 was downregulated in the meantime $(\mathrm{P}<0.05$, Figure 2). After treatment with IMD, it was observed that additional IMD decreased caspase- 3 activity and pro-apoptotic Bax expression, and increased the antiapoptotic $\mathrm{Bcl}-2$ protein levels compared with the respective hyperlipidemia animals $(\mathrm{P}<0.05$, Figure 2). These results demonstrated that IMD could improve myocardial MI/R injury by reduced cardiomyocyte apoptosis in hyperlipidemia animals.

\section{IMD attenuated MI/R-induced inflammation in hyperlipidemia rats}

To study the effects of inflammatory cytokines in hyperlipidemia rats treated with IMD during MI/R, we next investigated the level of TNF- $\alpha$ and IL- 6 in serum and myocardial tissues. MI/R significantly increased TNF- $\alpha$ and IL-6 levels compared to respective control groups $(\mathrm{P}<0.05$, Table 3$)$. Importantly, cytokine expression was significantly greater in hyper- 
lipidemia rats compared to non-hyperlipidemia ones, both in sham and $\mathrm{MI} / \mathrm{R}$ groups $(\mathrm{P}<0.05$, Table 3). Furthermore, in the IMD group, TNF- $\alpha$ and IL-6 levels were significantly reduced not only in serum but also the myocardial tissues $(\mathrm{P}<0.05$, Table 3$)$, suggesting that IMD administration at least partially decreased the inflammatory reaction in hyperlipidemia rats.
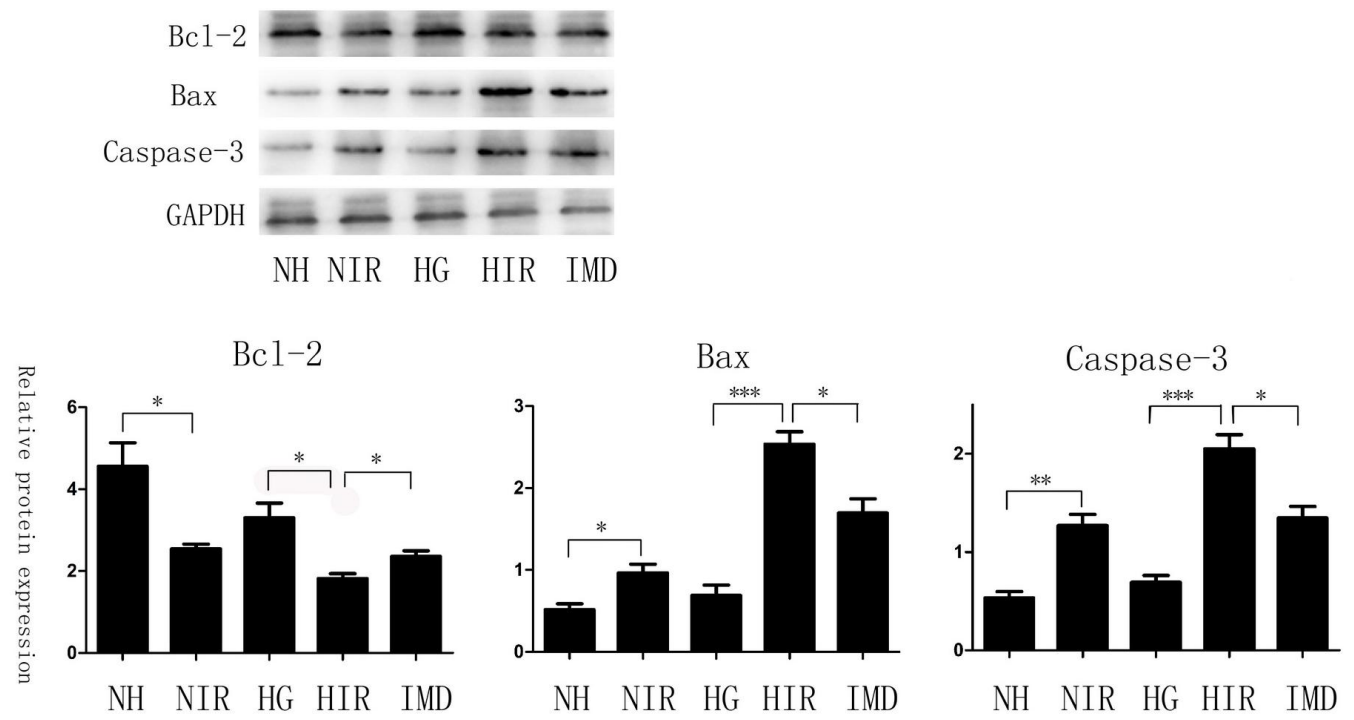

Figure 2. Intermedin (IMD) mitigates myocardial injury by reducing apoptosis. The activity of Caspase-3, Bcl-2, and Bax protein in myocardial tissues was shown by Western blot analysis. $\mathrm{NH}=$ non-hyperlipidemia sham; $\mathrm{NIR}=$ non-hyperlipidemia ischemia/reperfusion + vehicle; $\mathrm{HG}=$ hyperlipidemia sham; HIR = hyperlipidemia ischemia/ reperfusion + vehicle; IMD $=$ hyperlipidemia ischemia/reperfusion + IMD treatment. ${ }^{*} \mathrm{P}<0.05 ; * * \mathrm{P}<0.01 ; * * * \mathrm{P}$ $<0.001$ significant.

Table 3. Intermedin (IMD) decreases myocardial injury by reducing inflammatory reaction.

\begin{tabular}{|c|c|c|c|c|c|c|}
\hline \multicolumn{2}{|c|}{ Inflammatory marker (units)/group } & \multirow{2}{*}{$\frac{\mathrm{NH}}{81.54 \pm 6.88}$} & \multirow{2}{*}{$\frac{\text { NIR }}{173.62 \pm 8.51^{*}}$} & \multirow{2}{*}{$\frac{\mathrm{HG}}{107.36 \pm 5.39^{\mathbf{}}}$} & \multirow{2}{*}{$\frac{\text { HIR }}{209.76 \pm 7.47^{\Delta \Lambda}}$} & \multirow{2}{*}{$\frac{\text { IMD }}{174.11 \pm 8.04^{*}}$} \\
\hline Serum & $\mathrm{TNF}-\alpha(\mathrm{pg} / \mathrm{mL})$ & & & & & \\
\hline & IL-6 $(\mathrm{pg} / \mathrm{mL})$ & $109.64 \pm 5.98$ & $198.45 \pm 8.71^{*}$ & $134.55 \pm 6.32^{\nabla}$ & $280.07 \pm 9.30^{\Delta \Delta}$ & $225.52+8.33^{\star}$ \\
\hline \multirow[t]{2}{*}{ Myocardium } & TNF- $\alpha(\mathrm{pg} / \mathrm{mL})$ & $321.44 \pm 37.01$ & $615.37 \pm 21.74 *$ & $428.96 \pm 39.77^{\mathbf{}}$ & $912.58 \pm 41.23^{\Delta \boldsymbol{\Delta}}$ & $716.84 \pm 31.45^{*}$ \\
\hline & IL-6 $(\mathrm{pg} / \mathrm{mL})$ & $309.65 \pm 29.98$ & $655.79 \pm 32.03^{*}$ & $512.04 \pm 28.31^{\mathbf{V}}$ & $822.43 \pm 37.93^{\Delta \Delta}$ & $674.28 \pm 40.09^{*}$ \\
\hline
\end{tabular}

$\mathrm{NH}=$ non-hyperlipidemia sham; NIR = non-hyperlipidemia ischemia/reperfusion + vehicle; $\mathrm{HG}=$ hyperlipidemia sham; HIR = hyperlipidemia ischemia/reperfusion + vehicle; IMD $=$ hyperlipidemia ischemia/reperfusion + IMD treatment. Results are reported as means $\pm \mathrm{SE} .{ }^{*} \mathrm{P}<0.05$ vs $\mathrm{NH},{ }^{\nabla} \mathrm{P}<0.05 v s \mathrm{NH},{ }^{\Delta} \mathrm{P}<0.05$ vs $\mathrm{HG},{ }^{\Delta} \mathrm{P}<0.05 v s$ NIR, ${ }^{\bullet} \mathrm{P}<0.05$ vs HIR. $\mathrm{N}=10$ /group.

\section{DISCUSSION}

In the present study, we demonstrated that hyperlipidemia could significantly decrease the IMD level in a hyperlipidemia rat model. Moreover, we provided evidence that the elevated IMD levels after MI/R alleviated hyperlipidemia-mediated myocardium injury 
by reducing cardiomyocyte apoptosis and inflammatory reactions. The upregulation of IMD peptide in hyperlipidemia states may indicate an important role in myocardium protection against hyperlipidemia-mediated MI/R injury.

Cardiovascular disease is the principal cause of death in many countries, of which myocardial infarction is the most serious condition. In the variety of causes of MI, hyperlipidemia is a significant reason because it not only results in the deposition of lipids in the athermanous lesions but may produce the primary endothelial injury, which promotes the atherosclerotic process (Ross and Harker, 1976). Several large observational studies have assessed the association between blood lipid concentrations and cardiovascular risk. It was reported that both higher TG and TC levels were significant independent predictors of coronary death only in participants in the upper age range (Tverdal et al., 1989). After simultaneous adjustment for a variety of coronary risk factors, TG concentrations manifested significant correlation with MI, ischemic heart disease and survival after 26 years of follow-up in a large-scale prospective cohort study (Nordestgaard et al., 2007). Thus, improving the state in patients with high blood lipids may play an important role in ameliorating the quality of life and prognosis for coronary heart disease patients. Currently, our study first indicated that besides lipidlowering drugs such as statins, the newly discovered peptide intermedin also has a crucial role in the hyperlipidemia rat model. After $30 \mathrm{~min}$ MI and 120 min reperfusion, severe injury occurred in rat myocardium, along with increased LDH, CK-MB, MDA and decreased SOD levels, whereas IMD was shown to attenuate MI/R-induced injury by inducing the opposite changes. In addition, MDA is a well-accepted marker of oxidative stress, which reflects the extent of systemic lipid peroxidation. Moreover, the antioxidant SOD protects cells by reducing free radical-induced injury, whose levels reflect the body's capacity to scavenge oxygen free radicals (Rachmat et al., 2013; Yang et al., 2013). In our results, post-MI/R MDA levels were significantly increased in hyperlipidemia rats and markedly reduced by IMD treatment, while myocardial SOD activity was attenuated after MI/R and further upregulated in IMD group. These data demonstrated that IMD administration could partially reverse these outcomes, suggesting IMD may be involved in the inhibition of MI/R-induced oxidative stress in hyperlipidemia rats. Additionally, in view of the increased MDA content and decreased SOD activity observed in the hyperlipidemia MI/R group, we also suggest that hyperlipidemia-enhanced oxidative stress may exacerbate $\mathrm{MI} / \mathrm{R}$ injury.

Apoptosis is closely related to the occurrence and development of various diseases, while cardiomyocyte apoptosis contributes to a variety of heart diseases such as myocardial infarction, cardiomyopathy, progressive pump-failure and arrhythmias, as well as cardiac remodeling (Mehrhof et al., 2001). Numerous studies have shown that the Bcl-2 protein family plays an important role in the regulation of the apoptotic process, which could somehow cause diverse forms of intracellular damage and integrate the competing signals of death stimuli, and further determine cell survival (Adams and Cory, 1998; Babu et al., 2004). As a typical representative of this family, Bcl-2 has been reported to exert its anti-apoptotic effect by blocking the activation of caspase enzyme-associated signaling pathways, antagonizing the function of another family member Bax and so on (Adams and Cory, 1998; Henshall et al., 2002; Babu et al., 2004; Nieminen et al., 2013; Tinahones et al., 2013). Significantly, our study demonstrated that $\mathrm{MI} / \mathrm{R}$ increased caspase- 3 and Bax protein expression in hyperlipidemia animals, while the activity of Bcl-2 was decreased in the meantime, suggesting that hyperlipidemia rats showed exacerbated apoptosis after MI/R compared to non-hyperlipidemia rats. Moreover, 
previous studies have demonstrated that IMD administration decreases MI/R-induced cardiomyocyte apoptosis (Yang et al., 2005; Du et al., 2011), and that myocardial injury may be exacerbated by downregulated IMD during early reperfusion (Teng et al., 2013). Our research further reinforced these reports, which demonstrated that the alteration of apoptosis-associated genes after MI/R was attenuated by additional IMD treatment, indicating that IMD has an anti-apoptotic effect on the MI/R heart injury in hyperlipidemia models. Additionally, in our current study, inflammatory cytokines were shown to be regulated during MI/R and IMD administration was observed to decrease the hyperlipidemia-induced serum/myocardial TNF- $\alpha$ and IL-6 overexpression. These data provided a new clue to study the mechanism of IMD treatment for hyperlipidemia-associated myocardial injury, and thus, further investigations about whether IMD attenuates hyperlipidemia-induced MI/R injury via inflammatory signaling mitigation should be done.

\section{CONCLUSION}

In summary, our results indicated that IMD is involved in cardiac MI/R diseases. With additional IMD, MI/R injury of cardiomyocytes could be attenuated via reduction of apoptosis and inflammation in a hyperlipidemia rat model. Thus, IMD may be a potentially novel therapeutic target for ischemic injury of the hyperlipidemic heart, which warrants further studies.

\section{REFERENCES}

Adams JM and Cory S (1998). The Bcl-2 protein family: arbiters of cell survival. Science 281: 1322-1326.

Aronow HD, Lincoff AM, Quinn MJ, McRae AT, et al. (2008). Relation between previous lipid-lowering therapy and infarct size (creatine kinase-MB level) in patients presenting with acute myocardial infarction. Am. J. Cardiol. 102: $1119-1124$.

Assmann G and Schulte H (1992). Relation of high-density lipoprotein cholesterol and triglycerides to incidence of atherosclerotic coronary artery disease (the PROCAM experience). Prospective Cardiovascular Munster study. Am. J. Cardiol. 70: 733-737.

Babu PP, Suzuki G, Ono Y and Yoshida Y (2004). Attenuation of ischemia and/or reperfusion injury during myocardial infarction using mild hypothermia in rats: an immunohistochemical study of Bcl-2, Bax, Bak and TUNEL. Pathol. Int. 54: 896-903.

Bell D and McDermott BJ (2008). Intermedin (adrenomedullin-2): a novel counter-regulatory peptide in the cardiovascular and renal systems. Br. J. Pharmacol. 153 (Suppl 1): S247-S262.

Bell D, Zhao Y, McCoy FP, Devine A, et al. (2008). Expression of the counter-regulatory peptide intermedin is augmented in the presence of oxidative stress in hypertrophied cardiomyocytes. Cell Physiol. Biochem. 21: 409-420.

Chang CL, Roh J and Hsu SY (2004). Intermedin, a novel calcitonin family peptide that exists in teleosts as well as in mammals: a comparison with other calcitonin/intermedin family peptides in vertebrates. Peptides 25: 1633-1642.

Du QX, Yue W and Wang YY (2011). Effect and mechanism of intermedin in acute rat cardiac ischemic injury. Fa. Yi Xue Za Zhi 27: 164-168.

Fang J, Chen L, Wu L and Li W (2009). Intra-cardiac remote ischemic post-conditioning attenuates ischemia-reperfusion injury in rats. Scand. Cardiovasc. J. 43: 386-394.

Gao Y, Yao X, Zhang Y, Li W, et al. (2011). The protective role of hydrogen sulfide in myocardial ischemia-reperfusioninduced injury in diabetic rats. Int. J. Cardiol. 152: 177-183.

Henshall DC, Araki T, Schindler CK, Lan JQ, et al. (2002). Activation of Bcl-2-associated death protein and counterresponse of Akt within cell populations during seizure-induced neuronal death. J. Neurosci. 22: 8458-8465.

Jiang W, Jiang HF, Pan CS, Cai DY, et al. (2004). Relationship between the contents of adrenomedullin and distributions of neutral endopeptidase in blood and tissues of spontaneously hypertensive rats. Hypertens. Res. 27: 109-117.

Li H, Bian Y, Zhang N, Guo J, et al. (2013). Intermedin protects against myocardial ischemia-reperfusion injury in diabetic rats. Cardiovasc. Diabetol. 12: 91. 
Liu T, Yang LL, Zou L, Li DF, et al. (2013). Chinese medicine formula lingguizhugan decoction improves Beta-oxidation and metabolism of fatty acid in high-fat-diet-induced rat model of fatty liver disease. Evid. Based Complement. Alternat. Med. 2013: 429738.

Mehrhof FB, Muller FU, Bergmann MW, Li P, et al. (2001). In cardiomyocyte hypoxia, insulin-like growth factor-Iinduced antiapoptotic signaling requires phosphatidylinositol-3-OH-kinase-dependent and mitogen-activated protein kinase-dependent activation of the transcription factor cAMP response element-binding protein. Circulation 104: 2088-2094.

Nieminen AI, Eskelinen VM, Haikala HM, Tervonen TA, et al. (2013). Myc-induced AMPK-phospho p53 pathway activates Bak to sensitize mitochondrial apoptosis. Proc. Natl. Acad. Sci. U. S. A. 110: E1839-E1848.

Nordestgaard BG, Benn M, Schnohr P and Tybjaerg-Hansen A (2007). Nonfasting triglycerides and risk of myocardial infarction, ischemic heart disease, and death in men and women. JAMA 298: 299-308.

Osipov RM, Robich MP, Feng J, Clements RT, et al. (2009). Effect of thrombin fragment (TP508) on myocardial ischemiareperfusion injury in hypercholesterolemic pigs. J. Appl. Physiol. 106: 1993-2001.

Pan CS, Yang JH, Cai DY, Zhao J, et al. (2005). Cardiovascular effects of newly discovered peptide intermedin/ adrenomedullin 2. Peptides 26: 1640-1646.

Rachmat FD, Rachmat J, Sastroasmoro S and Wanandi SI (2013). Effect of allopurinol on oxidative stress and hypoxic adaptation response during surgical correction of tetralogy of fallot. Acta Med. Indones. 45: 94-100.

Roh J, Chang CL, Bhalla A, Klein C, et al. (2004). Intermedin is a calcitonin/calcitonin gene-related peptide family peptide acting through the calcitonin receptor-like receptor/receptor activity-modifying protein receptor complexes. J. Biol. Chem. 279: 7264-7274.

Ross R and Harker L (1976). Hyperlipidemia and atherosclerosis. Science 193: 1094-1100.

Smith RS, Jr., Gao L, Bledsoe G, Chao L, et al. (2009). Intermedin is a new angiogenic growth factor. Am. J. Physiol. Heart Circ. Physiol. 297: H1040-H1047.

Song Y, Zhang G, Zhu X, Pang Z, et al. (2012). Overexpression of the hydatidiform mole-related gene F10 inhibits apoptosis in A549 cells through downregulation of BCL2-associated X protein and caspase-3. Oncol. Lett. 4: 419-422.

Teng X, Bian Y, Cai Y, Duan X, et al. (2013). Downregulation of endogenous intermedin augmented myocardial injury in rats with ischemia/reperfusion. Horm. Metab. Res. 45: 206-212.

Tinahones FJ, Coin AL, Murri M, Oliva OW, et al. (2013). Caspase induction and BCL2 inhibition in human adipose tissue: a potential relationship with insulin signaling alteration. Diabetes Care 36: 513-521.

Tverdal A, Foss OP, Leren P, Holme I, et al. (1989). Serum triglycerides as an independent risk factor for death from coronary heart disease in middle-aged Norwegian men. Am. J. Epidemiol. 129: 458-465.

Wang TD, Wu CC, Chen WJ, Lee CM, et al. (1998). Dyslipidemias have a detrimental effect on left ventricular systolic function in patients with a first acute myocardial infarction. Am. J. Cardiol. 81: 531-537.

Wang TD, Chen WJ, Su SS, Lo SC, et al. (2002). Increased cardiomyocyte apoptosis following ischemia and reperfusion in diet-induced hypercholesterolemia: relation to Bcl-2 and Bax proteins and caspase-3 activity. Lipids 37: 385-394.

Yang JH, Qi YF, Jia YX, Pan CS, et al. (2005). Protective effects of intermedin/adrenomedullin2 on ischemia/reperfusion injury in isolated rat hearts. Peptides 26: 501-507.

Yang JH, Cai Y, Duan XH, Ma CG, et al. (2009). Intermedin 1-53 inhibits rat cardiac fibroblast activation induced by angiotensin II. Regul. Pept. 158: 19-25.

Yang Y, Duan W, Jin Z, Yi W, et al. (2013). JAK2/STAT3 activation by melatonin attenuates the mitochondrial oxidative damage induced by myocardial ischemia/reperfusion injury. J. Pineal Res. 55: 275-286.

Zhang HY, Jiang W, Liu JY, Li Y, et al. (2009). Intermedin is upregulated and has protective roles in a mouse ischemia/ reperfusion model. Hypertens. Res. 32: 861-868.

Zhang L, Ma J and Liu H (2012). Protective effect of ischemic postconditioning against ischemia reperfusion-induced myocardium oxidative injury in IR rats. Molecules 17: 3805-3817. 